\title{
Management of Dengue infection: improving morbidity
}

\author{
Vithanage Pujitha Wickramasinghe ${ }^{1}$ \\ ${ }^{1}$ Faculty of Medicine, University of Colombo, Sri Lanka
}

\author{
Article information \\ Total number of \\ Words 4100 \\ Author has no conflicts of interest to declare \\ Keywords: Dengue infection, antioxidants, \\ dengue vaccine, calcium supplement, \\ vitamin E supplement, $\mathrm{N}$-acetyle cyctine, \\ rupatadine
}

Date of submission: 10.06 .2019

Date of acceptance: 14.07 .2019

\author{
Author responsible for correspondence: \\ Prof. V. Pujitha Wickramasinghe \\ Department of Paediatrics \\ Faculty of Medicine, University of Colombo \\ Kynsey Road, Colombo, Sri Lanka \\ E-mail: pujithaw@yahoo.com
}

https://orcid.org/0000-0002-8355-1283

\begin{abstract}
Dengue is a vector borne disease, prevalent in the tropics in epidemic proportions. In recent years it has become one of the highest mortality causing communicable diseases in the tropics. Its pathophysiology is not completely understood. In the absence of a definitive treatment modality, supportive care is the most important tool. Having a better understanding of the haematological and biochemical changes is of paramount importance in order to provide effective supportive care to achieve speedy recovery with minimum morbidity and mortality. Although several candidate vaccines have been produced most are still undergoing phase III clinical trials.
\end{abstract}

This article intends to discuss identified and postulated pathophysiological modalities in dengue infection and possible therapeutic/management options that could be adopted to reduce dengue morbidity and mortality.

DOI: http://doi.org/10.4038/cjms.v56i1.4959

\section{Introduction}

Dengue fever is one of the most significant reemerging communicable diseases in the world involving tropical climates of South and Southeast Asia, the Caribbean and South America[1]. The epidemiological triad of dengue infections comprises of the AGENT, which is a single stranded enveloped RNA virus of the family of Flaviviridae, with antigenically distinct four serotypes, transmitted by the VECTOR Aedes mosquitoe, to the HOST, humans and monkeys, who amplifies the agent.

More than $40 \%$ of the world's population is now at risk from dengue. World Health Organization currently estimates between 50-100 million dengue infections worldwide each year[2]. According to data from the Epidemiology Unit of Sri Lanka, the total number of cases recorded each year up to 2017 was less than fifty thousand. However, an unprecedented number of cases amounting to more than 185,000 were reported in 2017. In 2018, once again, it was a little under fifty thousand with 52 deaths[3]. The highest case fatality rate for this country in 2009 was $1.0 \%$, but subsequently this came down to $0.2 \%$ even during the worst epidemic in 2017[3]. Many environmental factors such as water collection in disposed items and blocked drains, overcrowding and global warming have contributed to the increased transmission of disease. Global warming has shortened the extrinsic incubation period thus making the mosquito spread the virus rapidly. Further the enhanced life cycle, smaller size and relative dehydration has made the Aedies mosquito to bite frequently[4]. After an incubation period of 4-7 days (range 3-14) clinical symptoms arise[5]. Most dengue infections result in relatively mild illness, but some can progress to dengue haemorrhagic fever (DHF). DHF is a severe febrile disease characterized by abnormalities in haemostasis and increased 
vascular permeability where progression may result in dengue shock syndrome (DSS)[6].Tachycardia, hypotension and abdominal pain characterise the onset of plasma leakage (critical phase). DHF is always accompanied by a platelet count of $<100 \times 10^{9} / \mathrm{L}$, haemoconcentration (a rise in the packed cell volume $>20 \%$ of basal level), leucopenia $(<5 x$ $10^{9} / \mathrm{L}$ ), and raised liver enzymes[7]. When plasma leakage is severe, patients may develop other signs of circulatory disturbance such as prolonged capillary refill time and narrow pulse pressure, along with the development of pleural effusion and ascites[6,7].

Complications of dengue infection are usually associated with dengue haemorrhagic fever and dengue shock syndrome. Serious complications are hypotension, shock, thrombocytopenia, bleeding, liver and other organ damage. Metabolic complications include hypocalcaemia, hypoglycaemia, hyponatremia and acidosis. If untreated, mortality from the complications of dengue fever could be as high as $20 \%$, whereas if recognized early and managed properly, mortality could be less than $1 \%[8]$.

Dengue infection is difficult to be distinguished from the other viral infections. Traditionally diagnosis is based on serology. IgM against dengue can be detected after 5 days of fever using ELISA technique. Persistence of IgM in some patients in endemic areas makes it difficult to diagnose an acute infection[9]. Non structural protein-1 (NS1) is a glycoprotein secreted by cells infected by dengue virus and it has been used as an effective diagnostic tool[9]. It has a declining sensitivity from $90 \%$ to $84 \%$ during day 1 to 3 days after onset of illness with the decline of the viraemia[10]. Therefore it would be prudent to perform the assessment within first 48 hours of the fever to have a higher yield of positive results[8]. However, it cannot differentiate between those who progress to DF and DHF. Real time reverse transcriptase polymerase chain reaction (RT-PCR) is a highly sensitive diagnostic tool that can be used during acute infection but also with a decline in detection from $82 \%$ to $69 \%$ when performed during 1-3 days of onset of illness[9]. A single sample tested for both IgM and NS1 or RT-PCR between days 4-7 would detect $95-100 \%$ of cases[10]. However, the cost of molecular tests makes it an impractical tool in day-to-day clinical practice.

Dengue is potentially fatal unless managed appropriately and there is no definitive medication that could be used. Therefore, early diagnosis of dengue infection helps to strengthen monitoring and timely intervention, which is the key feature of management of dengue infection. Understanding the pathophysiology with early identification of biochemical and haematological changes, helps early intervention which reduce cellular damage thus reducing morbidity and case fatality.

\section{Pathogenesis}

With the inoculation of the virus by the mosquito, the virions bind to cell-surface receptors and are internalized through endocytosis and in the endosome the virion releases the RNA into the cytoplasm. Viral RNA is translated into a polyprotein and viral non-structural protein replication occurs. Virus now assembles in the endoplasmic reticular (ER) membrane, where capsid protein and viral RNA are enveloped by the ER membrane and glycoproteins to form immature virus particles which are transported through the secretory pathway before mature viruses are released[11].

Several hypotheses for the pathogenesis of the clinical spectrum of dengue virus infection have been proposed. Among them, antibody-dependent enhancement (ADE) of infection has long been thought to play a central role[7]. After the first infection with a dengue virus (primary infection), body develops non-neutralizing cross reactive antibodies against other three serotypes and subsequent infection with one of them (secondary infection) leads to effective internalization of virus into mononuclear cells. It will rapidly increase the viral load causing more severe disease(DHF) due to intense immune activation[12]. The aberrant immune responses not only delay virus clearance, but also trigger cytokine overproduction and autoantibodies to platelets and endothelial cells that initiate subsequent dysfunction of these cells. Cytokines contribute to the evolution of the disease from an asymptomatic state to severe form of dengue. Cytokines released by these cells and activated $\mathrm{T}$ cells cause endothelial dysfunction leading to increased vascular permeability and vascular leak, the hallmark of DHF. This leads to development of pleural effusion, ascites and shock[13]. A mathematical model had been developed to predict development of DHF looking at a combination of cytokines[14].

Evidence demonstrates that oxidative stress plays an important role in dengue infection[15], similar to other viral infections such as HIV, Viral Hepatitis[16] and Influenza[17]. When infected 
with a dengue virus, host immune system gets activated and reactive oxygen species (ROS) are produced in the neutrophils, monocytes and endothelial cells. Levels of hydroxyeicosatetraenoic acid products (HETEs), F2-isoprostanes (F2-IsoPs), and cholesterol oxidation products (COPs) have been assessed in different stages of dengue infection as markers of oxidative stress[18]. Levels of HETEs and F2IsoPs were higher during the febrile phase compared to the convalescent level. The level of oxidative stress was maximum in DSS followed by DHF and was minimal in DF $[18,19]$.

These ROS are removed by antioxidants like Vitamins (C, E, A), glutathione and enzymes (superoxide dismutase and glutathione peroxidase) The oxidative stress occurs when the amount of oxidants exceeds the concentration of antioxidants[18]. This ultimately result in organ damage and increased risk of secondary infection[20]. Additionally, in vitro studies done on human liver cells infected with dengue virus have shown evidence of oxidative damage[21].

\section{Liver injury}

Liver cell damage and dysfunction in dengue viral hepatitis results in elevated liver enzymes and coagulopathy. Dengue virus replicates in both hepatocytes and Kupffer cells[22]. Liver injury in dengue viral infection occurs through several mechanisms. (a) Host defenses operating thorough cytokines causing cellular damage; (b) Immune injury through CD8 cells recognizing viral antigens on hepatocytes; (c) Viral cytopathy where the virus directly induces hepatocyte apoptosis, and (d) Endothelial injury caused by Anti-NS-1 antibodies cross reacting with endothelial antigens[23].

\section{Haematological changes}

In most cases of DF, haematological parameters are normal. The main haematological abnormalities are leucopenia and thrombocytopenia caused by direct destructive action of the dengue virus[24]. A Sri Lankan study showed that leucocyte count and platelet count on day 2.5 as predictors of entry into critical phase[25]. Although the predictive power was not as high as some of the biochemical markers, a platelet count $<100 \times 10^{9} / \mathrm{L}$ had a sensitivity of $76.9 \%$ and a specificity of $79.3 \%$ while total leucocyte count $<2.6 \times 10^{9} / \mathrm{L}$ had a sensitivity of $69.2 \%$ and a specificity of $82.8 \%$. Lowest platelet counts were observed on day 4.5 in DHF (Median
$-35 \times 10^{9} / \mathrm{L}, \mathrm{IQR} 25.75-44.28 \times 10^{9} / \mathrm{L}$ ) and on day 6.5 in DF (Median $-72.5 \times 10^{9} / \mathrm{L}$, IQR 55.0 $97.25 \times 10^{9} / \mathrm{L}$ ). Increase in haematocrit closely reflected the decline of the platelet count in both DF and DHF, which was more distinct and rapid in the latter. The highest haematocrit of DHF was seen on day 4, which denotes the onset of the critical phase[25].

\section{Biochemical changes}

Series of biochemical changes are observed during the course of the illness and they can be used to predict the course of illness and development of complications.

\section{Changes in serum calcium}

Hypocalcaemia occurs during the leaking phase of individuals and correction improves the outcome. Hypocalcaemia is common in DHF and it could cause convulsions. Furthermore there are reports that oral supplementation reduces the disease burden[26]. Calcium has also been implicated in the immuopathogenesis of dengue, however the precise clinical implications of these interactions are not yet clearly defined. Derangements of calcium homeostasis are likely to be associated with myocardial dysfunction and platelet aggregation. Hypocalcaemia occurs during the leaking phase of individuals and correction improves the outcome[26].

A study done on adults at Teaching Hospital Anuradhapura showed that none of the patients with normocalcaemia developed significant fluid leak. Mean serum ionized calcium levels among patients with third space leak was 0.97 (SD 0.1) $\mathrm{mmol} / \mathrm{l}$ compared to $1.12(\mathrm{SD} \quad 0.14) \mathrm{mmol} / \mathrm{l}$ among those without fluid leak $(\mathrm{p}=0.035)$. Patients with hypocalcaemia had significantly lower platelet count and serum albumin levels[27]. A study done among Sri Lankan children did not show any distinct pattern of change in calcium levels [25]. This could be due to early administration of oral calcium lactate, $1 \mathrm{mmol} / \mathrm{kg} / \mathrm{d}$, for all suspected cases of dengue fever as per study protocol. In spite of supplementation with calcium lactate, there was no increase in serum calcium during the acute phase, but once acute phase was over, levels began to rise. This indicates that these patients may have leaked out calcium during acute phase and supplementation would have maintained normocalcaemia [25]. Based on this evidence, the author routinely supplement all suspected patients with dengue infection, with $10 \mathrm{mg} / \mathrm{kg}$ of elemental 
calcium twice daily, irrespective of the calcium status, and it could be one reason for low morbidity and mortality rates observed. However, its effect on the natural course on the illness needs to be evaluated.

\section{Changes in serum albumin and cholesterol}

During the leaking phase albumin and cholesterol levels also decline. Serum albumin of $<3.5 \mathrm{~g} / \mathrm{dL}$ or a drop of $>0.5 \mathrm{~g} / \mathrm{dL}$ and serum cholesterol of $<100 \mathrm{mg} / \mathrm{dL}$ or a drop of $>20 \mathrm{mg} / \mathrm{dL}$ suggest entry into critical phase[6]. Data from Sri Lanka showed that in DHF, serum albumin and cholesterol showed a decline with the increase in the haematocrit. In DHF patients, serum albumin showed a negative correlation with the haematocrit from day 3 to day 6 and was significant on day $4(\mathrm{r}=0.49, \mathrm{p}=0.006)$ and day 4.5 $(r=0.41, p=0.022)$ [25]. The best cut off value of serum albumin to predict entry into critical phase was $37.5 \mathrm{~g} / \mathrm{L}$ (sensitivity of $86.7 \%$; specificity of $77.8 \%)[25]$.

Plasma lipid profile differs according to disease severity and serum cholesterol, HDL, and LDL levels could be used as potential predictors of clinical outcome[28]. Lipids regulate cytokine levels and modify the host immune response, while cytokines modify lipid metabolism. Triglycerides increase with severity of infection, probably due to increase in lipolysis and de novo fatty acid synthesis in the liver[28]. Reduction in the level of serum cholesterol was seen between $3^{\text {rd }}$ and $4^{\text {th }}$ day of illness and the cut off value to predict entry into critical phase was a reduction of serum cholesterol of $0.38 \mathrm{mmol} / \mathrm{L}$ between day 3 and 4 (sensitivity of $77.3 \%$; specificity of $71.9 \%)[25]$.

\section{Changes in liver enzymes}

Hepatic dysfunction is a well-recognized complication, even going on to fulminant hepatic failure, of dengue infections demonstrated by hepatomegaly and increased transaminase levels $[29,30]$. Alanine transaminase(ALT), Aspartate transaminase(AST), Alkaline phosphatise(ALP) levels were significantly higher in patients with DHF compared to DF [31]. In a series of 61 children (2 months to 12 years), $87 \%$ of children with hepatomegaly and $81 \%$ without hepatomegaly had raised transaminase levels[31]. Enzyme levels increased during the latter half of the illness. DSS and DHF had significantly higher levels compared to DF. Therefore monitoring changes in AST, ALT and ALP would be useful in identifying the presence and extent of hepatic involvement. In dengue infection, elevations in serum AST appear to be greater than ALT levels and transaminases levels[22, 32]. This differs from the pattern in viral hepatitis, in which ALT levels are usually higher than or equal to AST levels. In children with non-alcoholic fatty liver disease the elevation of ALT would be higher than AST.

Elevated AST levels tend to return to normal more rapidly than ALT levels. This is possibly because AST has a shorter half-life (12.5 - 22 hours) than ALT (32 - 43 hours)[22]. Elevated transaminase levels return to normal by $14-21$ days after infection. Skeletal muscle injury could also contribute to elevated AST levels. A steep rise in transaminases during first seven days, would suggest significant liver damage, which would be a deviation from the normal course of liver damage seen in DF. In very severe cases of dengue infection AST and ALT could rise over $1000 \mathrm{U} / \mathrm{L}$, and in such instances close observation for liver dysfunction (encephalopathy, coagulopathy) as well as other organ dysfunction is very important[23]. It is important to balance fluid meticulously during such times as under perfusion as well as fluid overload leading to liver engorgement could aggravate tissue hypoxia in liver. Increased levels of ALP and bilirubin were found in a smaller proportion of cases[33].

A study done in Sri Lanka on 5-12 year old children showed that both ALT and AST levels increased significantly between day 3 and 4 and reached peak concentration during the later stages. Median concentration of AST at the peak was 746 U/L(IQR 215-1011) and ALT was 118 U/L(IQR 110 - 314). Serum AST levels have remained higher than ALT throughout the illness in both DF and DHF[25].

\section{Clinical management of dengue infection}

Case management includes the differentiation of DF and DHF based on plasma leakage as the discriminating factor rather than haemorrhage. Management of DHF included identification of the beginning and predicting the end of the critical (plasma leakage) phase, meticulous monitoring, accurate fluid management, organ support, correction of metabolic derangement, early detection and treatment of concealed bleeding and other complications.

\section{- Onset of leaking}

- Rise of capillary Packed Cell Volume (PCV) $>20 \%$ from base line 
- Pleural effusion or ascites in ultrasound scan

- Fall in serum albumin by $0.5 \mathrm{~g} / \mathrm{dl}$ or

- Fall in serum cholesterol by $20 \mathrm{mg} / \mathrm{dl}$

- End of leaking

- Beginning of rise of platelets

- Reduction in fluid requirement

- Acral itchiness

- Establishment of diuresis

- Wide splitting of second heart sound

If a battery of tests could be used to predict the onset of complications early in the course of the illness, it would help the clinician to design effective and timely managements plans to improve morbidity and mortality[6].

\section{Liver injury}

Understanding the multiple mechanisms of liver injury seen in DF should allow the exploration of different modes of treatment options. N-acetyl cysteine (NAC) is one such that has been used in severe liver disease although there are no specific trials done against dengue infection[23, 34]. The author's experience in this treatment modality, a dose of $20-30 \mathrm{mg} / \mathrm{kg}$ in $2-3$ divided doses orally, is useful in halting the progression of liver damage, thus reversing the rise of liver transaminases when specially used at the early stages with the onset of rapid rise $(\times 10$ of transaminases normal) of transaminases. In severe form of liver derangement, intravenous NAC, $100 \mathrm{mg} / \mathrm{kg}$ dissolved in $5 \%$ dextrose to prepare $48 \mathrm{ml}$ of solution is infused over 24 hours. Depending on response several cycles could be used. There are other potential treatment modalities available in research stage targeting stages of viral binding to hepatocytes and inhibition of signaling pathways mediating apoptosis[35].

\section{Controlling the oxidative stress}

Despite increasing evidence that oxidative stress is an important pathological process in the disease, studies evaluating the efficacy of antioxidant treatment are scarce. The sole interventional study conducted by Vaish et al, only focused on the platelet count of adult dengue patients who received vitamin $\mathrm{E}$ treatment during the illness[36]. This finding suggests that vitamin $E$ treatment reduces the bone marrow effects of the dengue virus and also helps in faster recovery. Vitamin E as an antioxidant, acts as a peroxyl radical scavenger, preventing the propagation of free radicals in tissues, by reacting with them to form a tocopheryl radical, which will then be reduced by a hydrogen donor and thus return to its reduced state[37]. In addition to being an antioxidant Vitamin $\mathrm{E}$ is also involved in immune function, enzyme activation and gene expression[38].

Side effects of Vitamin E supplementation are minimal. No syndrome of acute vitamin E toxicity has been described. The upper daily limits in children are $200 \mathrm{mg}$ at 1 to 3 years, $300 \mathrm{mg}$ at 4 to 8 years and $600 \mathrm{mg}$ at 9 to 13 years[39]. Nogueira et al[20] demonstrated that supplementing Vitamin $\mathrm{E}$ and $\mathrm{C}$ were able to reduce lipid peroxidation in critically ill patients and to decrease the number of deaths and days of mechanical ventilation. Vaish and others administered vitamin E (400mg) to adult patients with DF to study the effect on thrombocytopenia[36]. A significant increase in platelet count was observed in the vitamin $\mathrm{E}$ group.

A randomized controlled study done in Sri Lanka among children has shown that duration of leaking is significantly reduced by vitamin $\mathrm{E}$ supplementation (33.5 hours vs 44.8 hours; $\mathrm{p}=0.023)$ [40]. In vitamin E supplemented group serum albumin was higher, haematocrit, was lower suggesting that there was less leakage [40]. Furthermore, mean leukocyte count and platelet count were higher in the vitamin E group although statistically this was not significant. This study also showed that the mean AST and ALT levels were lower in the vitamin E group compared to placebo.

\section{Newer therapeutic modalities in secondary prevention}

Newer agents have been studied at present based on known pathophysiological effects. Plateletactivating factor (PAF) is a lipid mediator released by mast cells and eosinophils in humans. Its action in the development of allergic diseases is well known[41]. It had been noted that PAF is elevated during acute dengue infection and may be an important mediator in plasma leak. Therefore blocking the PAF could lead to reduction in severity of leak. Rupatadine, an $\mathrm{H}_{1}$ receptor antagonist is used for the treatment of allergic conditions. Rupatadine has been identified to have an inhibitory action on PAF receptors as well[41]. Therefore rupatadine might modulate dengue-associated vascular leak acting through PAF receptors. A randomised, placebocontrolled trial in 183 adult patients in Sri Lanka with acute dengue, showed that rupatadine up to 
$40 \mathrm{mg}$ daily appeared to be safe and well-tolerated with minimum adverse events[42]. Higher platelet counts and lower transaminase levels on day 7 were seen in the rupatadine group compared to the placebo. Authors concluded that the potential beneficial effect of rupatadine requires further evaluation in large studies focused on recruitment during the early febrile phase[42].

Understanding the changes in biochemical and haematological parameters during a dengue infection and also identifying the place of administration of different medications in the management of dengue infection is important. Some of the research prospects in areas of secondary prevention are; sparing use of fluid (half bolus concept, oral bolus); active fluid removal (early use of oral furosemide); use of NAC during early onset of liver injury; preventing gastropathy and bleeding (routine use of proton pump inhibitors); supplementation of calcium; reducing viral load (antivirals, host targets to reduce replication -kinases and glucosidase inhibitors); prevent/treat severe disease (modulate cytokine release and vascular leak, antioxidants).

\section{Primary prevention}

This would be the best method to control such communicable disease. With the excellent track record Sri Lanka has in control of vaccine preventable diseases, a robust vaccine would be the answer to this illness.

The natural immunity developed through natural exposure to bites of mosquitoes carrying dengue viruses are only partially understood. It is being complicated by the inter-relationship between the host and the four distinct serotypes of the virus. Dengue virus infection induces high-titers of neutralizing antibodies which could impart a protective immune response[43]. After the first dengue infection (primary infection) with a particular dengue virus serotype, long lasting protection occurs against that serotype (homotypic protection). It is also understood that these antibodies could provide cross-protection against the other 3 serotypes (heterotypic protection), which could last over 2 years[44]. Therefore window of cross-protection induced by a first infection against the second symptomatic infection is about 2 years.

It is well accepted that following waning off of cross-neutralizing antibodies, second illness is more likely to be a severe illness with a second dengue virus infection than with the first[45]. Following recovery from the second infection, neutralizing antibodies are made in a broader scale resulting in less severe infection (multitypic protection) in subsequent infections. The mechanisms causing severe infection with the second virus infection is not well understood [44].

Challenge in developing a vaccine against dengue are; existence of four serotypes, pre-existing immunity in the community, seasonality of the transmission with changes in the prevalence of the serotype and possible genetic preponderance to severity of illness. Currently several dengue vaccines are undergoing phase III clinical trials. A mathematical model developed on dengue transmission in Thailand, has helped to evaluate the usefulness of different vaccination strategies[46].

The first vaccine to receive approval was the recombinant live attenuated tetravalent CYDTDV vaccine (Dengvaxia ${ }^{\circledR}$, Sanofi Pasture, France). It is a yellow fever vaccine virus backbone vector that expresses envelope proteins of dengue viruses type 1 to 4[47]. However the vaccine demonstrated partial efficacy against dengue infection[48]. Vaccine efficacy at the end of 25 months post-enrolment were $60.3 \%$ (95\% CI, 55.7 to 64.5 ) for all participants, $65.6 \%$ (95\% CI, 60.7 to 69.9) for those 9 years of age or older, and $44.6 \%(95 \% \mathrm{CI}, 31.6$ to 55.0$)$ for those younger than 9 years of age[48]. Efficacy in children under 9 years was lower[48]. Several countries licensed and used this vaccine. However, some safety concerns arose when reanalyzing data from the follow up period in the third-year post vaccine. In the 2-5-year age group (in the Asian cohort), there was a higher incidence of hospitalizing dengue illnesses, with a relative risk of 7.7 compared to the placebo. Therefore the manufacturer recommended the vaccine only for children above 9 years of age[48].

WHO in 2016, issued a position paper and outlined recommendations for the use of the dengue vaccine in areas with high disease burden[5]. WHO recommended to use the CYDTDV where sero-prevalence in the target age group is $>70 \%$. In populations with $50 \%$ and $70 \%$ it is acceptable but the impact may be lower. It is not recommended when the sero-prevalence is $<50 \%$. If CYD-TDV is introduced, it should be administered as a 3 -dose series given on a 0/6/12 month schedule.

Takeda ${ }^{\circledR}$ Japan is currently working on a tetravalent candidate, Tetravalent Dengue Vaccine (TDV), includes a whole attenuated DEN2 virus and recombinant DEN1, DEN3, and DEN4 using the DEN2 backbone. Currently it is 
undergoing phase III trials in eight countries in Asia and Latin America region due to end in December 2021.

Dengue vaccine should only be a part of a comprehensive dengue control strategy, which should also include broad and sustainable vector control programme, periodically updated, evidence-based clinical care, and an extensive dengue surveillance system[5]. Introduction of the vaccine is a country level decision considering local priorities, national and regional dengue epidemiology, and the cost-effectiveness. WHO recommends countries to have well-organized pharmaco-vigilance system with the capacity to monitor and manage adverse reactions following immunization[5]. Countries planning to introduce vaccination should have a good reporting system on hospitalized patients with adequate data on different clinical stages of dengue infections.

\section{Conclusion}

Dengue, a viral infection, is a self limiting illness with no specific medication but varied degree of morbidity and mortality which could be altered with close monitoring and supportive care. Understanding the underlying pathophysiology would help to identify possible supportive therapeutic and management modalities to improve the clinical outcome, till an effective vaccine is developed.

\section{References}

1. Jeremy JW. Dengue. British Medical Bulletin 2010;95:13.

2. WHO. Dengue and severe dengue factsheet (revised in January 2012). Wkly Epidemiol Rec. 2012;87(8):68-70.

3. Epidemiology Unit MoH. Disease Surveillance Trends. 2019.

4. Lee H, Kim JE, Lee S, Lee CH. Potential effects of climate change on dengue transmission dynamics in Korea. PLoS One. 2018;13(6):e0199205.

5. WHO. Dengue vaccine: WHO position paper - July 2016. Wkly Epidemiol Rec. 2016;91(30):349-64.

6. Epidemiology Unit MoH. Sri Lanka National guidelines on management of Dengue fever and Dengue haemorrhagic fever in children and adolescent. 2012.

7. Lei HY, Yeh TM, Liu HS, Lin YS, Chen $\mathrm{SH}$, Liu CC. Immunopathogenesis of dengue virus infection. J Biomed Sci. 2001;8(5):377-88.
8. WHO. WHO report on global surveillance of epidemic-prone infectious diseases. Geneva: World Health Organization; 2000. Report No.: WHO/CDS/CSR/ISR/2000.1.

9. Whitehorn J, Farrar J. Dengue. Br Med Bull. 2010;95:161-73.

10. Hunsperger EA, Munoz-Jordan J, Beltran $\mathrm{M}$, Colon C, Carrion J, Vazquez J, et al. Performance of Dengue Diagnostic Tests in a Single-Specimen Diagnostic Algorithm. J Infect Dis. 2016;214(6):83644.

11. Perera R, Khaliq M, Kuhn RJ. Closing the door on flaviviruses: entry as a target for antiviral drug design. Antiviral Res. 2008;80(1):11-22.

12. Halstead SB. Pathogenesis of dengue: challenges to molecular biology. Science. 1988;239(4839):476-81.

13. Malavige GN, Ogg G. Pathogenesis of severe dengue infection. Ceylon Med J. 2012;57(3):97-100.

14. Jayasundara SD, Perera SS, Malavige GN, Jayasinghe $\mathrm{S}$. Mathematical modelling and a systems science approach to describe the role of cytokines in the evolution of severe dengue. BMC Syst Biol. 2017;11(1):34.

15. Seet RC, Lee CY, Lim EC, Quek AM, Yeo LL, Huang SH, et al. Oxidative damage in dengue fever. Free Radic Biol Med. 2009;47(4):375-80.

16. Stehbens WE. Oxidative stress in viral hepatitis and AIDS. Exp Mol Pathol. 2004;77(2):121-32.

17. Akaike T, Noguchi Y, Ijiri S, Setoguchi K, Suga M, Zheng YM, et al. Pathogenesis of influenza virus-induced pneumonia: involvement of both nitric oxide and oxygen radicals. Proc Natl Acad Sci U S A. 1996;93(6):2448-53.

18. Gil L, Martinez G, Tapanes R, Castro O, Gonzalez D, Bernardo L, et al. Oxidative stress in adult dengue patients. Am J Trop Med Hyg. 2004;71(5):652-7.

19. Soundravally R, Sankar P, Bobby Z, Hoti SL. Oxidative stress in severe dengue viral infection: association of thrombocytopenia with lipid peroxidation. Platelets. 2008;19(6):447-54.

20. Nogueira CR, Borges F, Lameu E, Franca C, Ramalho A. Effects of supplementation of antioxidant vitamins and lipid peroxidation in critically ill patients. Nutr Hosp. 2013;28(5):1666-72.

21. Lin YL, Liu CC, Chuang JI, Lei HY, Yeh $\mathrm{TM}$, Lin YS, et al. Involvement of oxidative stress, NF-IL-6, and RANTES expression in dengue-2-virus-infected human liver cells. Virology. 2000;276(1):114-26. 
22. Seneviratne SL, Malavige GN, de Silva HJ. Pathogenesis of liver involvement during dengue viral infections. Trans R Soc Trop Med Hyg. 2006;100(7):608-14.

23. Dissanayake HA, Seneviratne SL. Liver involvement in dengue viral infections. Rev Med Virol. 2018;28(2).

24. Kalayanarooj S, Vaughn DW, Nimmannitya S, Green S, Suntayakorn S, Kunentrasai N, et al. Early clinical and laboratory indicators of acute dengue illness. J Infect Dis. 1997;176(2):313-21.

25. Kularatnam GAM, Jasinge E, Gunasena S, Samaranayake D, Senanayake MP, Wickramasinghe VP. Evaluation of biochemical and haematological changes in dengue fever and dengue hemorrhagic fever in Sri Lankan children: a prospective follow up study. BMC Pediatr. 2019;19(1):87.

26. Sanchez-Valdez E, Delgado-Aradillas M, Torres-Martinez JA, Torres-Benitez JM. Clinical response in patients with dengue fever to oral calcium plus vitamin D administration: study of 5 cases. Proc West Pharmacol Soc. 2009;52:14-7.

27. Dahanayaka NJ, Agampodi SB, Kodithuwakku Arachchi UP, Vithange SP, Rajapakse R, Ranathunga K, et al. Dengue fever and ionized calcium levels: significance of detecting hypocalcaemia to predict severity of dengue. Ceylon Med J. 2017;62(1):67-9.

28. van Gorp ECM, Suharti, C., Mairuhu, A.T,A., Dolmans, W.M.V., van der Ven, V., Demacker, P.N.M., van der Meer J.W.M. Changes in the Plasma Lipid Profile as a Potential Predictor of Clinical Outcome in Dengue Hemorrhagic Fever. Clinical Infectious Diseases. 2002;34:4.

29. Lim G, Lee JH. N-acetylcysteine in children with dengue-associated liver failure: a case report. J Trop Pediatr. 2012;58(5):409-13.

30. Trung DT, Thao le TT, Hien TT, Hung NT, Vinh NN, Hien PT, et al. Liver involvement associated with dengue infection in adults in Vietnam. Am J Trop Med Hyg. 2010;83(4):774-80.

31. Mohan B, Patwari AK, Anand VK. Hepatic dysfunction in childhood dengue infection. J Trop Pediatr. 2000;46(1):40-3.

32. Kuo CH, Tai DI, Chang-Chien CS, Lan CK, Chiou SS, Liaw YF. Liver biochemical tests and dengue fever. Am J Trop Med Hyg. 1992;47(3):265-70.

33. Gulati S, Maheshwari A. Atypical manifestations of dengue. Trop Med Int Health. 2007;12(9):1087-95.
34. Sklar GE, Subramaniyam, M. Acetyl cysteine treatment for non-acetaminopheninduced acute liver failure. . Annals of Pharmacotherapy. 2004;38:3.

35. Sreekanth GP, Chuncharunee A, Sirimontaporn A, Panaampon J, Noisakran S, Yenchitsomanus PT, et al. SB203580 Modulates p38 MAPK Signaling and Dengue Virus-Induced Liver Injury by Reducing MAPKAPK2, HSP27, and ATF2 Phosphorylation. PLoS One. 2016;11(2):e0149486.

36. Vaish A, Verma, S., Agarwal, A., Gupta, L., Gutch, M.. Effect of vitamin $\mathrm{E}$ on thrombocytopenia in dengue fever. Annals of Tropical Medicine and Public Health 2012;5:4.

37. Traber MG, Stevens JF. Vitamins C and E: beneficial effects from a mechanistic perspective. Free Radic Biol Med. 2011;51(5):1000-13.

38. NIH. Vitamin E: Fact sheet for professionals. USA: Office of Dietary supplements, National Institutes of Health; [Availablefrom:

http://ods.od.nih.gov/factsheets/VitaminE HealthProfessional.

39. Medicine Io. Dietary Reference Intakes for Vitamin C, Vitamin E, Selenium, and Carotenoids. Washington (DC)2000.

40. Prasad Chathurangana PW, Samaranayake, D.B.D.L, Quienters, V.G, Wickramasinghe, V.P. Effects of vitamin E supplementation on the clinical outcome of dengue fever and dengue haemorrhagic fever in children. Asian Pacific Journal of Tropical Disease. 2017;7(11):5.

41. Munoz-Cano R, Ainsua-Enrich E, TorresAtencio I, Martin M, Sanchez-Lopez J, Bartra J, et al. Effects of Rupatadine on Platelet- Activating Factor-Induced Human Mast Cell Degranulation Compared With Desloratadine and Levocetirizine (The MASPAF Study). J Investig Allergol Clin Immunol. 2017;27(3):161-8.

42. Malavige GN, Wijewickrama A, Fernando S, Jeewandara C, Ginneliya A, Samarasekara S, et al. A preliminary study on efficacy of rupatadine for the treatment of acute dengue infection. Sci Rep. 2018;8(1):3857.

43. Rodrigo WW, Block OK, Lane C, Sukupolvi-Petty S, Goncalvez AP, Johnson $\mathrm{S}$, et al. Dengue virus neutralization is modulated by IgG antibody subclass and Fcgamma receptor subtype. Virology. 2009;394(2):175-82.

44. Montoya M, Gresh L, Mercado JC, Williams KL, Vargas MJ, Gutierrez G, et 
al. Symptomatic versus inapparent outcome in repeat dengue virus infections is influenced by the time interval between infections and study year. PLoS Negl Trop Dis. 2013;7(8):e2357.

45. Endy TP, Yoon IK, Mammen MP. Prospective cohort studies of dengue viral transmission and severity of disease. Curr Top Microbiol Immunol. 2010;338:1-13.

46. Chao DL, Halstead SB, Halloran ME, Longini IM, Jr. Controlling dengue with vaccines in Thailand. PLoS Negl Trop Dis. 2012;6(10):e1876.

47. WHO. GACVS Statement on Dengvaxia ${ }^{\circledR}$ (CYD-TDV). Global Vaccine Safety. 2017.

48. Hadinegoro SR, Arredondo-Garcia JL, Capeding MR, Deseda C, Chotpitayasunondh $\mathrm{T}$, Dietze $\mathrm{R}$, et al. Efficacy and Long-Term Safety of a Dengue Vaccine in Regions of Endemic Disease. N Engl J Med. 2015;373(13):1195-206. 